\title{
A New Efficient Convergent Synthesis of Conjugated Aryl-containing Dendrimers
}

\author{
Ibrahim Mustafa El-Deeb ${ }^{\dagger, t}$ and So Ha Lee ${ }^{\dagger, *}$ \\ ${ }^{\dagger}$ Life/Health Division, Korea Institute of Science and Technology, P.O. Box 131, Seoul 130-650, Korea \\ *E-mail:LSH6211@kist.re.kr \\ Department of Biomolecular Science, University of Science and Technology, Daejeon 305-333, Korea \\ Received February 26, 2010, Accepted March 26, 2010
}

Key Words: Dendrimers, Convergent synthesis, Photoluminescence, Suzuki coupling

\begin{abstract}
Dendrimers are branched macromolecules that consist of a core, one or more dendrons, and surface groups. Due to their controlled mass, uniform structure, surface functionality and good solubility (relative to linear polymers), they have been studied for a wide range of pharmaceutical applications including solubility enhancement, ${ }^{1,2}$ drug delivery,${ }^{3,4}$ MRI contrast agents, ${ }^{5,6}$ neutron capture therapy, ${ }^{7}$ gene therapy ${ }^{8}$ and photodynamic therapy. ${ }^{9}$ At the same time, conjugated dendrimers have recently attracted special attention for their potential use in organic light emitting diodes, either as light emitting or charge-
\end{abstract} transporting layers. ${ }^{10,11}$

The unique structure of dendrimers allowed for better and more flexible control of their properties. The fact that different parts of dendrimer structure can have different functions allows for independent optimization of the electronic and processing properties. For example, the core in dendrimer can be selected to give the desired luminescence color, while the surface groups could be controlled to give the desired solubility. ${ }^{10}$ Despite the large amount of research focused at dendrimers and the large number of prospects for their potential uses, very few dendrimer-based products have reached an industrial development stage. Among the reasons for the slow progress in this area are the multi-step synthetic routes commonly employed for the synthesis of dendrimers and the purification processes required to remove the excess of reagents and byproducts at each generation build-up. Accordingly, much effort have been devoted to develop synthetic methodologies that either follow a growing pattern from a multivalent core in an interactive sequence (divergent), or a dendron is grafted on to the core (convergent). Some progress has been made in this regard, including the polyamidoamine (PAMAM) dendrimers reported by Tomalia, ${ }^{12}$ phosphorus-containing dendrimers by Majoral and co-workers, ${ }^{13}$ aliphatic polyester dendrimers by Fréchet and co-workers, ${ }^{14}$ and the 3,5-dihydroxybenzyl alcohol based dendrimers by Bourrier and Kakkar. ${ }^{15}$ A number of click chemistry protocols have been also applied recently for the preparation of dendrimers, employing Diels-Alder reaction, ${ }^{16}$ thiol-ene reactions ${ }^{17}$ and azide-alkyne reactions. ${ }^{18,19}$ However, there are still needs to make these processes viable for industrial scale applications.

Herein we describe a new convergent synthesis of conjugated dendrimers starting with acetyl substituted dendrons which were dehydrated under the effect of tetrachlorosilane to form a 1,3,5-trisubstituted phenyl core carrying three branches (dendrons) in a star shaped dendrimer. ${ }^{20}$ This resulted dendrimer was then subjected to a series of divergent Suzuki couplings for functionalization of the final dendrimers with different groups. ${ }^{21}$ The synthetic protocol is simple and could be easily modified to obtain either conjugated or saturated dendrimers bearing variable groups. The products obtained in each preparation step are easily purified by simple crystallization, making the process easily applicable at larger industrial scale.

As shown in Scheme 1, the synthesis of the key dendron 2 started by the Suzuki coupling of 4-bromotriphenylamine with 4-acetylphenylboronic acid under the catalysis of dichlorobis(triphenylphosphine) $\mathrm{Pd}(\mathrm{II})$ and in the presence of sodium carbonate in a mixed solvent of acetonitrile and water in a $(1: 1, \mathrm{v} / \mathrm{v})$ ratio. The resulted highly green luminescent acetyl intermediate 1 was obtained in a good yield of $76 \%$, and was then subjected to $p$-phenyl bromination under the effect of $N$-bromosuccinimide in DMF to yield the target dibromoacetyl-dendron $\mathbf{2}$ in 97\% yield. Every three moles of the key dendron 2 were then dehydrated at the acetyl-moiety terminal part under the effect of excess tetrachlorosilane to yield the hexabromo intermediate dendrimer $\mathbf{3}$ in a $72 \%$ yield. This intermediate dendrimer $\mathbf{3}$ was then subjected to a series of Suzuki coupling reactions with a number of phenyl boronic acids in the presence of dichlorobis(triphenylphosphine)Pd(II) and potassium carbonate in a mixed solvent of THF/water in a $(4: 1, \mathrm{v} / \mathrm{v})$ ratio to yield the final target dendrimers 4a-i in moderate to good yields.

In order to get a more clear picture about the effect of substitution at these new conjugated dendrimers on their photophysical properties, the absorption and photoluminescence (PL) spectra of compounds $\mathbf{4 a - i}$ were recorded in dichloromethane using UV-vis. and luminescence spectroscopy, respectively. The results are shown in Table 1 and the typical spectra are shown in Figure 1.

As shown in Figure 1, the UV spectra of all the recorded compounds showed absorption maxima in the range of (350 $380 \mathrm{~nm}$ ). Excitation of these compounds by a single beam UV light at the $\lambda_{\max }$ of their absorption spectra resulted in an intense emission at the range of $(450-510 \mathrm{~nm})$ at a very low concentration of $10 \mathrm{nM}$. By correlating between compounds structures and the intensity of photoluminescence, we found that the substitution with an electron deficient heterocyclic system like the pyridine ring in $\mathbf{4 b}$ reduced the luminescence intensity. The $m$-substitution pattern (as in $\mathbf{4 e}$ and $\mathbf{4} \mathbf{g}$ ) was found also to be unfavored for good luminescence. However, the $p$-substitution (as in 4f) remains to be the best for the highest luminescence. 

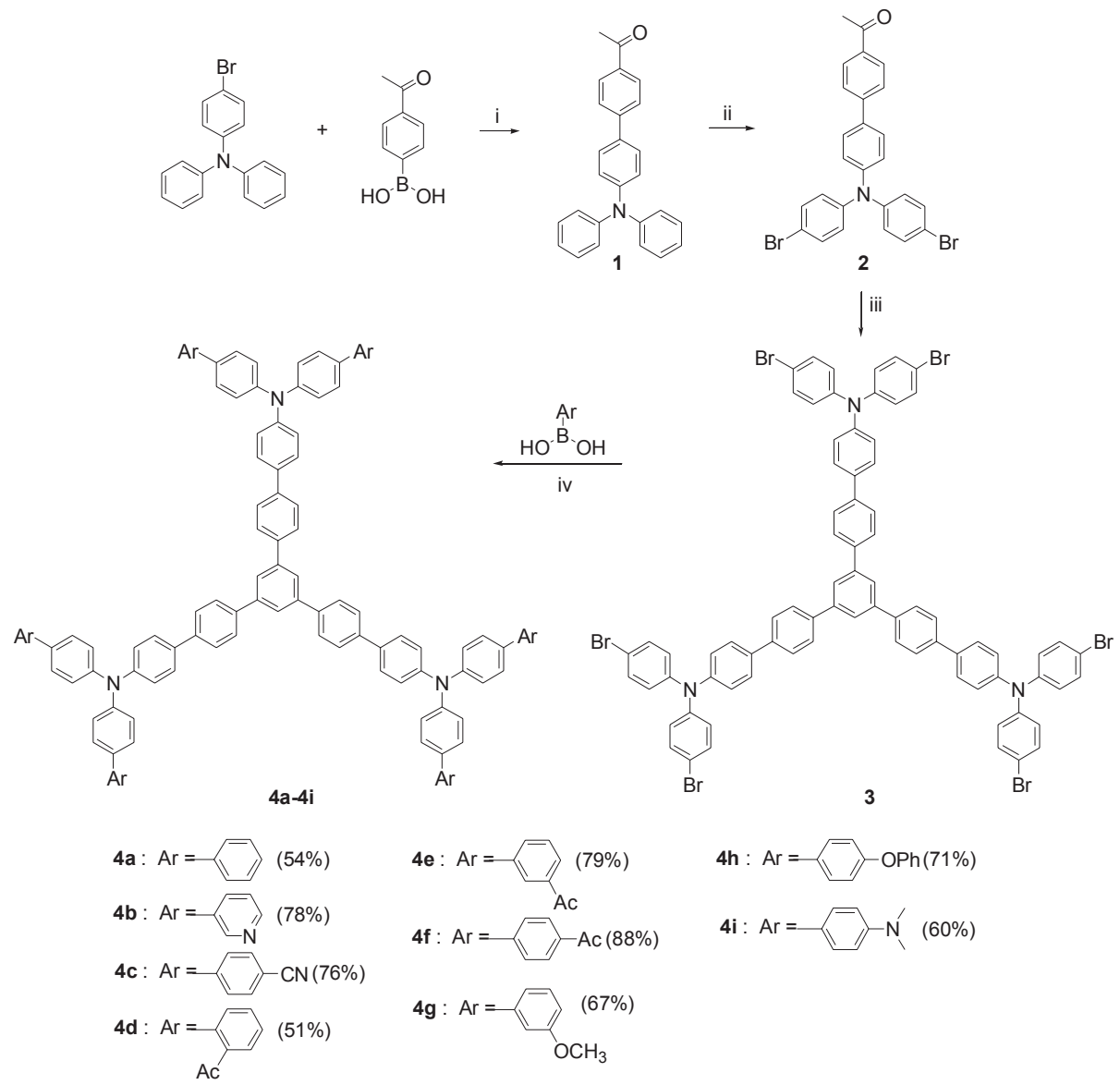

Reaction conditions and yields: (i) $\mathrm{Pd}\left(\mathrm{PPh}_{3}\right)_{2} \mathrm{Cl}_{2}, \mathrm{Na}_{2} \mathrm{CO}_{3}, \mathrm{~N}_{2}, \mathrm{CH}_{3} \mathrm{CN} / \mathrm{H}_{2} \mathrm{O}(1: 1, \mathrm{v} / \mathrm{v}), 80^{\circ} \mathrm{C}, 3 \mathrm{~h}, 76 \%$ (ii) NBS, DMF, $60^{\circ} \mathrm{C}$, $18 \mathrm{~h}, 97 \%$ (iii) $\mathrm{SiCl}_{4}$, EtOH/toluene (5:1, v/v), reflux, $24 \mathrm{~h}, 72 \%$ (iv) $\mathrm{Pd}_{(}\left(\mathrm{PPh}_{3}\right)_{3} \mathrm{Cl}_{2}, \mathrm{~K}_{2} \mathrm{CO}_{3}, \mathrm{~N}_{2}, \mathrm{THF} / \mathrm{H}_{2} \mathrm{O}(4: 1), 80^{\circ} \mathrm{C}, 24 \mathrm{~h}$.

Scheme 1. Synthetic pathway for dendrimers 4a-i and reaction conditions

Table 1. The absorption and luminescence maxima of the compounds 4a-i

\begin{tabular}{cccc}
\hline \multirow{2}{*}{ Compound } & \multicolumn{2}{c}{$\lambda_{\max }(\mathrm{nm})$} & FWHM $^{c}(\mathrm{~nm})$ \\
\cline { 2 - 3 } & $\mathrm{UV}^{a}$ & $\mathrm{PL}^{b}$ & \\
\hline $\mathbf{4 a}$ & 353 & 468 & 87 \\
$\mathbf{4 b}$ & 355 & 489 & 115 \\
$\mathbf{4} \mathbf{c}$ & 371 & 484 & 78 \\
$\mathbf{4 d}$ & 354 & 503 & 85 \\
$\mathbf{4 e}$ & 358 & 501 & 101 \\
$\mathbf{4 f}$ & 373 & 508 & 91 \\
$\mathbf{4 g}$ & 355 & 459 & 70 \\
$\mathbf{4 h}$ & 355 & 491 & 91 \\
$\mathbf{4 i}$ & 358 & 500 & 91 \\
\hline
\end{tabular}

measured in $\mathrm{CH}_{2} \mathrm{Cl}_{2}$ solution at $5 \mu \mathrm{M}$ concentration, ${ }^{b}$ recorded by single beam excitation in $\mathrm{CH}_{2} \mathrm{Cl}_{2}$ solution at $10 \mathrm{nM}$ concentration, ${ }^{c}$ The full widths at half maximum value.

In summary, we have designed a new simple and efficient convergent synthetic protocol for the preparation of conjugated dendrimers. The synthetic pathway could be modified easily for the preparation of different conjugated and saturated suitably substituted dendrimers for variable applications. The synthetic advantages and the ease of purification by simple crystalliza- tion allows for the large scale application of this synthetic protocol at the industrial scale.

\section{Experimental Section}

General. ${ }^{1} \mathrm{H}-\mathrm{NMR}(300 \mathrm{MHz})$ and ${ }^{13} \mathrm{C}-\mathrm{NMR}(75 \mathrm{MHz})$ were recorded on a Bruker Avance 300 spectrometer with TMS as an internal reference. The IR spectra were recorded on Perkin Elmer Spectrum GX spectrometer. Melting points were taken on a Thomas-Hoover capillary melting apparatus and were uncorrected. UV-visible spectra and luminescence spectra were recorded on S-2130 spectroscopy and RF-5301-PC luminescent spectroscopy, respectively. Column chromatography was performed on Merck silica gel 60 (230 - 400 mesh). TLC was carried out using glass sheets precoated with silica gel 60 F254 prepared by E. Merck. All the commercially available reagents were obtained from Aldrich and Tokyo Kasei Chemical and generally used without further purification.

1-(4'-Diphenylaminobiphenyl-4-yl)ethanone (1): A mixture of 4-bromotriphenylamine (2.0 g, $6.17 \mathrm{mmol})$, 4-acetylphenylboronic acid $(1.12 \mathrm{~g}, 6.80 \mathrm{mmol})$, dichlorobis(triphenylphosphine)Pd(II) (130 mg, $0.19 \mathrm{mmol}$ ) and $\mathrm{Na}_{2} \mathrm{CO}_{3}(460 \mathrm{mg}, 4.32$ $\mathrm{mmol}$ ) was stirred in a mixture of acetonitrile and water (1:1, $\mathrm{v} / \mathrm{v}, 80 \mathrm{~mL}$ ). $\mathrm{N}_{2}$ gas was bubbled into this mixture for $10 \mathrm{~min}$, 

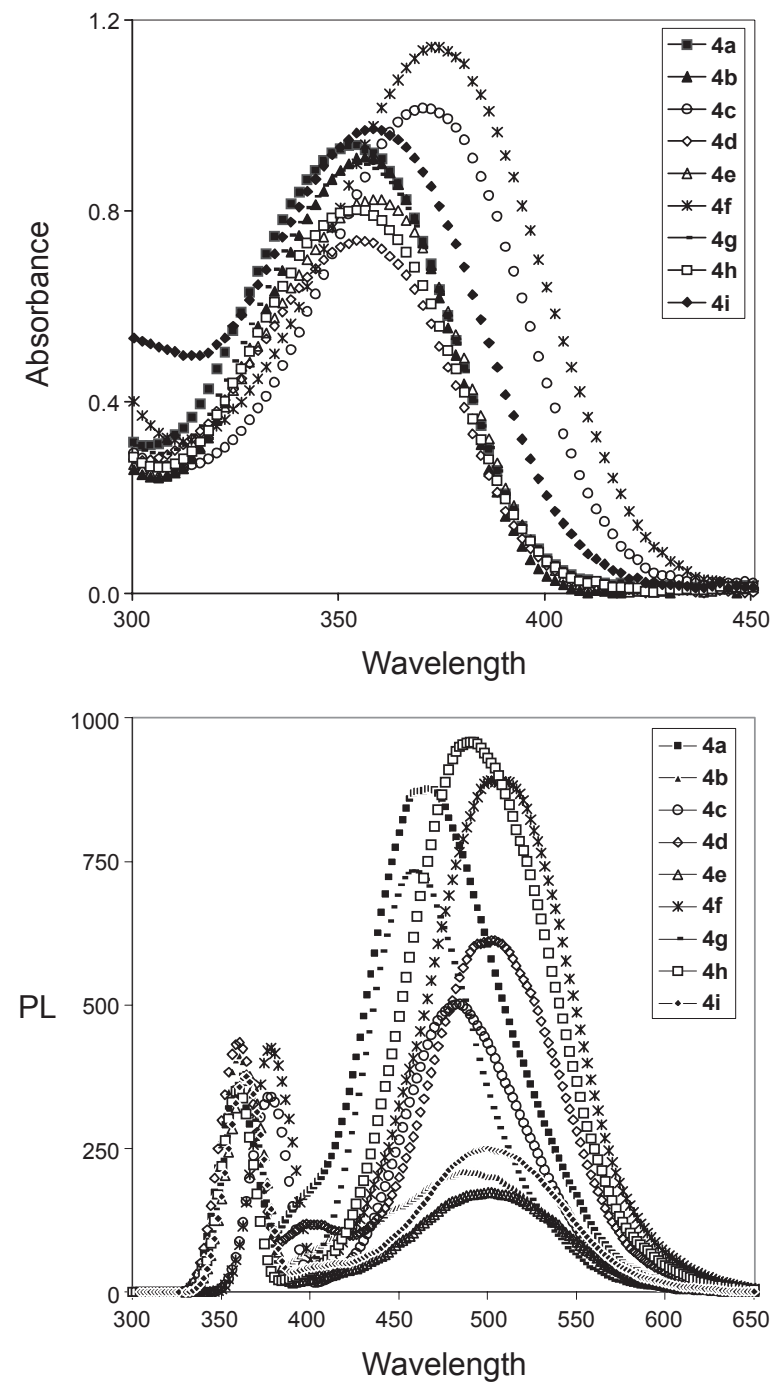

Figure 1. UV(upper pannel) and PL(lower pannel) spectra for compounds 4 a-i.

and then the mixture was heated at $80{ }^{\circ} \mathrm{C}$ while stirring under $\mathrm{N}_{2}$ atmosphere for $3 \mathrm{~h}$. The reaction mixture was left to cool at room temperature, poured into ice water $(80 \mathrm{~mL})$, and then extracted with methylene chloride $(150 \mathrm{~mL} \times 3)$. The organic layer was separated, dried over anhydrous $\mathrm{MgSO}_{4}$, and then evaporated under vacuum to yield the crude product which was then purified by flash chromatography (silica gel, hexane : EtOAc $=6: 1, \mathrm{v} / \mathrm{v})$ to give $1(1.7 \mathrm{~g}, 76 \%$ yield $)$ as bright green powder. mp $97-98{ }^{\circ} \mathrm{C}$; IR (KBr) v 1679, 1590, 1489, 1268, $821,750,697 \mathrm{~cm}^{-1} ;{ }^{1} \mathrm{H}-\mathrm{NMR}\left(300 \mathrm{MHz}, \mathrm{CDCl}_{3}\right) \delta 2.65(\mathrm{~s}, 3 \mathrm{H})$, $7.08(\mathrm{t}, J=7.3 \mathrm{~Hz}, 2 \mathrm{H}), 7.15-7.33(\mathrm{~m}, 10 \mathrm{H}), 7.53(\mathrm{~d}, J=8.5$ $\mathrm{Hz}, 2 \mathrm{H}), 7.68$ (d, $J=8.2 \mathrm{~Hz}, 2 \mathrm{H}), 8.03(\mathrm{~d}, J=8.1 \mathrm{~Hz}, 2 \mathrm{H})$; ${ }^{13} \mathrm{C}-\mathrm{NMR}\left(75 \mathrm{MHz}, \mathrm{CDCl}_{3}\right) \delta$ 26.64, 123.29, 123.37, 124.79, $126.50,127.93,128.98,129.39,133.12,135.32$, 145.22, 147.42, $148.19,197.71$.

1-(4'-(, $\mathrm{N}$-Bis(4-bromophenyl)amino)biphenyl-4-yl)ethanone (2): A mixture of compound 1 (1.5 g, $4.13 \mathrm{mmol})$ and $\mathrm{N}$-bromosuccinimide $(1.55 \mathrm{~g}, 8.67 \mathrm{mmol})$ was stirred in DMF $(10 \mathrm{~mL})$ at room temperature for $1 \mathrm{~h}$, then heated at $60^{\circ} \mathrm{C}$ for $18 \mathrm{~h}$. The reaction mixture was left to cool, then poured over brine solution containing ice $(200 \mathrm{~mL})$. The resulted precipitate was filtered, washed with cold water, dried, and then crystallized from ethylacetate to yield pure 2 (1.85 g, 97\% yield) as yellow crystals. mp $181-182^{\circ} \mathrm{C}$; IR (KBr) v 1680, 1602, 1486, $1313,1286,1266,820 \mathrm{~cm}^{-1}$; ${ }^{1} \mathrm{H}-\mathrm{NMR}\left(300 \mathrm{MHz}, \mathrm{CDCl}_{3}\right) \delta 2.65$ $(\mathrm{s}, 3 \mathrm{H}), 7.01(\mathrm{~d}, J=8.8 \mathrm{~Hz}, 4 \mathrm{H}), 7.14(\mathrm{~d}, J=8.6 \mathrm{~Hz}, 2 \mathrm{H}), 7.39$ $(\mathrm{d}, J=8.8 \mathrm{~Hz}, 4 \mathrm{H}), 7.54(\mathrm{~d}, J=8.6 \mathrm{~Hz}, 2 \mathrm{H}), 7.66(\mathrm{~d}, J=8.4$ $\mathrm{Hz}, 2 \mathrm{H}), 8.03$ (d, $J=8.4 \mathrm{~Hz}, 2 \mathrm{H}) ;{ }^{13} \mathrm{C}-\mathrm{NMR}\left(75 \mathrm{MHz}, \mathrm{CDCl}_{3}\right)$ $\delta 26.65,116.14,124.00,125.93,126.64,128.25,129.01,132.54$, $134.44,135.59,144.66,146.15,147.15,197.63$.

1,3,5-Tris(4'-( $N, N$-bis(4-bromophenyl)amino)biphenyl-4yl)benzene (3): To a stirred suspension of 2 (1.5 g, $2.88 \mathrm{mmol})$ in a mixture of abs. ethanol and dry toluene $(5: 1, \mathrm{v} / \mathrm{v}, 30 \mathrm{~mL})$ was added silicon tetrachloride $(6.6 \mathrm{~mL}, 57.6 \mathrm{mmol})$ dropwise under $\mathrm{N}_{2}$ atmosphere. The reaction temperature was raised to reflux, and the mixture was heated under $\mathrm{N}_{2}$ for $24 \mathrm{~h}$. The reaction mixture was left to cool, and the resulted green precipitate was filtered, washed with cold ethanol, and crystallized from chloroform/ethanol mixture, to yield pure 3 ( $1.1 \mathrm{~g}, 72 \%$ yield $)$ as yellowish green crystals. $\mathrm{mp}>300{ }^{\circ} \mathrm{C}$; IR $(\mathrm{KBr})$ v 1578 , 1483, 1312, 1270, 1070, 1005, 817, $511 \mathrm{~cm}^{-1}$; ${ }^{1} \mathrm{H}-\mathrm{NMR}(300$ $\left.\mathrm{MHz} \mathrm{CDCl}_{3}\right) \delta 7.02(\mathrm{~d}, J=8.7 \mathrm{~Hz}, 12 \mathrm{H}), 7.15(\mathrm{~d}, J=8.4 \mathrm{~Hz}$, $6 \mathrm{H}), 7.39(\mathrm{~d}, J=8.7 \mathrm{~Hz}, 12 \mathrm{H}), 7.57(\mathrm{~d}, J=8.5 \mathrm{~Hz}, 6 \mathrm{H}), 7.71(\mathrm{~d}$, $J=8.1 \mathrm{~Hz}, 6 \mathrm{H}), 7.80(\mathrm{~d}, J=8.2 \mathrm{~Hz}, 6 \mathrm{H}), 7.89(\mathrm{~s}, 3 \mathrm{H}),{ }^{13} \mathrm{C}-\mathrm{NMR}$ $\left(75 \mathrm{MHz}, \mathrm{CDCl}_{3}\right) \delta 115.80,124.43,124.90,125.70,127.18$, $127.77,128.02$, 132.27, 132.47, 135.57, 139.63, 139.74, 141.94, 146.34 .

General procedure for the synthesis of compounds $4 a-4 i$. A mixture of compound 3 (200 $\mathrm{mg}, 0.13 \mathrm{mmol}$ ), the appropriate aryl boronic acid derivative $(1.06 \mathrm{mmol})$, dichlorobis(triphenylphosphine)Pd(II) (28 mg, $0.04 \mathrm{mmol})$ and $\mathrm{K}_{2} \mathrm{CO}_{3}(110 \mathrm{mg}$, $0.80 \mathrm{mmol})$ was stirred in a mixture of THF and water $(4: 1$, $\mathrm{v} / \mathrm{v}, 10 \mathrm{~mL}$ ). $\mathrm{N}_{2}$ gas was bubbled into this mixture for $10 \mathrm{~min}$, and then the mixture was heated at $80{ }^{\circ} \mathrm{C}$ while stirring under $\mathrm{N}_{2}$ atmosphere for $24 \mathrm{~h}$. The reaction mixture was left to cool at room temperature, poured into brine solution $(50 \mathrm{~mL})$, and then extracted with methylene chloride $(100 \mathrm{~mL} \times 3)$. The organic layer was separated, dried over anhydrous $\mathrm{MgSO}_{4}$, and then evaporated under vacuum to yield the crude product which was then purified by crystallization from the appropriate solvent.

1,3,5-Tris(4'-( $N, N$-bis(biphenyl-4-yl)amino)biphenyl-4-yl) benzene (4a): It was obtained from toluene as light brown powder (107 mg, 54\% yield); mp $207-208{ }^{\circ} \mathrm{C}$; IR (KBr) v 1599, 1517, 1485, 1321, 1292, 817, 763, $696 \mathrm{~cm}^{-1}$; ${ }^{1} \mathrm{H}-\mathrm{NMR}$ (300 MHz, $\left.\mathrm{CDCl}_{3}\right) \delta 7.03-7.33(\mathrm{~m}, 18 \mathrm{H}), 7.39(\mathrm{~d}, J=6.9 \mathrm{~Hz}, 6 \mathrm{H}), 7.47-7.52$ $(\mathrm{m}, 12 \mathrm{H}), 7.59-7.67(\mathrm{~m}, 30 \mathrm{H}), 7.78(\mathrm{~d}, J=7.6 \mathrm{~Hz}, 6 \mathrm{H}), 7.85(\mathrm{~d}$, $J=7.6 \mathrm{~Hz}, 6 \mathrm{H}), 7.95(\mathrm{~s}, 3 \mathrm{H}) ;{ }^{13} \mathrm{C}-\mathrm{NMR}\left(75 \mathrm{MHz} \mathrm{CDCl}_{3}\right) \delta$ $124.38,124.53,125.70,126.74,126.95,127.14,127.75,127.85$, 127.96, 128.80, 134.91, 135.74, 139.86, 140.59, 141.98, 146.80, $147.03 ; \mathrm{UV} \lambda_{\max }=353 \mathrm{~nm}\left(\mathrm{CH}_{2} \mathrm{Cl}_{2}\right) ; \mathrm{PL} \lambda_{\max }=468 \mathrm{~nm}\left(\mathrm{CH}_{2} \mathrm{Cl}_{2}\right)$.

1,3,5-Tris (4'-( $N, N$-bis(4-(pyridin-3-yl)phenyl)amino)biphenyl-4-yl)benzene (4b): It was obtained from DMSO as buff powder (155 mg, 78\% yield); mp $239-240{ }^{\circ} \mathrm{C}$; IR (KBr) v $1599,1513,1496,1474,1322,1291,1269,817,801,710,557$ $\mathrm{cm}^{-1}$; ${ }^{1} \mathrm{H}-\mathrm{NMR}\left(300 \mathrm{MHz}, \mathrm{CDCl}_{3}\right) \delta$ 7.29-7.39 (m, 24H), 7.54 $(\mathrm{d}, J=7.9 \mathrm{~Hz}, 12 \mathrm{H}), 7.63(\mathrm{~d}, J=7.8 \mathrm{~Hz}, 6 \mathrm{H}), 7.74(\mathrm{~d}, J=7.6$ $\mathrm{Hz}, 6 \mathrm{H}), 7.82$ (d, J=7.6 Hz, 6H), 7.87-7.91 (m, 9H), 8.59 (d, $J=2.6 \mathrm{~Hz}, 6 \mathrm{H}), 8.88(\mathrm{~s}, 6 \mathrm{H})$; UV $\lambda_{\max }=355 \mathrm{~nm}\left(\mathrm{CH}_{2} \mathrm{Cl}_{2}\right)$; PL 
$\lambda_{\max }=489 \mathrm{~nm}\left(\mathrm{CH}_{2} \mathrm{Cl}_{2}\right)$.

$1,3,5$-Tris $(4$ '-( $N, N$-bis(4'-cyanobiphenyl-4-yl)amino)biphenyl-4-yl)benzene (4c): It was obtained from DMSO as greenish yellow powder ( $166 \mathrm{mg}, 76 \%$ yield); $\mathrm{mp} 250-252{ }^{\circ} \mathrm{C}$; IR (KBr) v 2226, 1596, 1493, 1324, 1292, 1184, 817, $533 \mathrm{~cm}^{-1}$; ${ }^{1} \mathrm{H}-\mathrm{NMR}\left(300 \mathrm{MHz}, \mathrm{CDCl}_{3}\right) \delta 7.28(\mathrm{~d}, J=8.7 \mathrm{~Hz}, 18 \mathrm{H}), 7.56$ $(\mathrm{d}, J=8.6 \mathrm{~Hz}, 12 \mathrm{H}), 7.63-7.77(\mathrm{~m}, 36 \mathrm{H}), 7.83(\mathrm{~d}, J=8.1 \mathrm{~Hz}$, 6H), 7.91 (s, 3H); ${ }^{13} \mathrm{C}-\mathrm{NMR}\left(75 \mathrm{MHz}, \mathrm{CDCl}_{3}\right) \delta 110.45,119.05$, $124.33,124.93,125.30,127.11,127.24,127.81,128.10,128.21$, $132.68,133.50,135.99,139.65,139.80,141.95,144.82,146.34$, 147.78; $\mathrm{UV} \lambda_{\max }=371 \mathrm{~nm}\left(\mathrm{CH}_{2} \mathrm{Cl}_{2}\right) ; \mathrm{PL} \lambda_{\max }=484 \mathrm{~nm}\left(\mathrm{CH}_{2} \mathrm{Cl}_{2}\right)$.

1,3,5-Tris (4'-( $N, N$-bis (2'-acetylbiphenyl-4-yl)amino)biphenyl-4-yl)benzene (4d): It was purified by flash chromatography (silica gel, hexane : $\mathrm{EtOAc}=1: 1, \mathrm{v} / \mathrm{v}$ ) to afforded the target compound as golden yellow powder ( $118 \mathrm{mg}, 51 \%$ yield); mp $161-162{ }^{\circ} \mathrm{C}$; IR (KBr) v 1686, 1599, 1509, 1496, 1474 , $1321,1266,1232,817,761 \mathrm{~cm}^{-1} ;{ }^{1} \mathrm{H}-\mathrm{NMR}\left(300 \mathrm{MHz}, \mathrm{CDCl}_{3}\right)$ $\delta 2.19(\mathrm{~s}, 18 \mathrm{H}), 7.25-7.67(\mathrm{~m}, 60 \mathrm{H}), 7.76(\mathrm{~d}, J=8.1 \mathrm{~Hz}, 6 \mathrm{H})$, $7.85(\mathrm{~d}, J=8.0 \mathrm{~Hz}, 6 \mathrm{H}), 7.94(\mathrm{~s}, 3 \mathrm{H}) ;{ }^{13} \mathrm{C}-\mathrm{NMR}(75 \mathrm{MHz}$, $\left.\mathrm{CDCl}_{3}\right) \delta 30.54,124.06,125.03,127.25,127.31,127.82,127.98$, $128.10,129.99,130.14,130.84,132.06,132.19,135.25,135.68$, $139.74,140.08,140.88,141.99,146.61,147.18,204.91 ; \mathrm{UV}$ $\lambda_{\max }=354 \mathrm{~nm}\left(\mathrm{CH}_{2} \mathrm{Cl}_{2}\right) ; \mathrm{PL} \lambda_{\max }=503 \mathrm{~nm}\left(\mathrm{CH}_{2} \mathrm{Cl}_{2}\right)$.

1,3,5-Tris (4'-( $N, N$-bis (3'-acetylbiphenyl-4-yl)amino)biphenyl-4-yl)benzene (4e): It was obtained from toluene as yellow powder ( $183 \mathrm{mg}, 79 \%$ yield); $\mathrm{mp} 176-178^{\circ} \mathrm{C}$; IR (KBr) v 1685, 1599, 1514, 1496, 1479, 1323, 1295, 1236, 818, 795, $693 \mathrm{~cm}^{-1} ;{ }^{1} \mathrm{H}-\mathrm{NMR}\left(300 \mathrm{MHz}, \mathrm{CDCl}_{3}\right) \delta 2.68$ (s, $\left.18 \mathrm{H}\right), 7.29$ $(\mathrm{d}, J=8.7 \mathrm{~Hz}, 18 \mathrm{H}), 7.51-7.64(\mathrm{~m}, 24 \mathrm{H}), 7.74(\mathrm{~d}, J=8.2 \mathrm{~Hz}$, $6 \mathrm{H}), 7.81(\mathrm{~d}, J=7.7 \mathrm{~Hz}, 12 \mathrm{H}), 7.90-7.94(\mathrm{~m}, 9 \mathrm{H}), 8.22(\mathrm{~s}, 6 \mathrm{H})$; ${ }^{13} \mathrm{C}-\mathrm{NMR}\left(75 \mathrm{MHz}, \mathrm{CDCl}_{3}\right) \delta 26.77,124.51,124.70,124.87$, $126.47,126.93$, 127.16, 127.77, 127.95, 128.10, 129.10, 131.26, $134.69,135.35,137.71,139.68,139.74,141.07,141.95,146.75$, $147.21,198.09 ; \mathrm{UV} \lambda_{\max }=358 \mathrm{~nm}\left(\mathrm{CH}_{2} \mathrm{Cl}_{2}\right) ; \mathrm{PL} \lambda_{\max }=501 \mathrm{~nm}$ $\left(\mathrm{CH}_{2} \mathrm{Cl}_{2}\right)$.

1,3,5-Tris (4'-( $N, N$-bis (4'-acetylbiphenyl-4-yl)amino)biphenyl-4-yl)benzene (4f): It was obtained from toluene as orange powder (205 mg, $88 \%$ yield); $\mathrm{mp} 255-257^{\circ} \mathrm{C}$; IR (KBr) v 1682, 1596, 1522, 1493, 1324, 1268, 817, $598 \mathrm{~cm}^{-1} ;{ }^{1} \mathrm{H}-\mathrm{NMR}$ $\left(300 \mathrm{MHz}, \mathrm{CDCl}_{3}\right) \delta 2.65(\mathrm{~s}, 18 \mathrm{H}), 7.28(\mathrm{~d}, J=7.6 \mathrm{~Hz}, 18 \mathrm{H})$, $7.58-7.75(\mathrm{~m}, 36 \mathrm{H}), 7.81(\mathrm{~d}, J=8.0 \mathrm{~Hz}, 6 \mathrm{H}), 7.90(\mathrm{~s}, 3 \mathrm{H}), 8.04$ $(\mathrm{d}, J=8.1 \mathrm{~Hz}, 12 \mathrm{H}) ;{ }^{13} \mathrm{C}-\mathrm{NMR}\left(75 \mathrm{MHz}, \mathrm{CDCl}_{3}\right) \delta 26.70$, $124.35,125.03,126.62,127.20,127.79,128.01,128.20,129.05$, $134.20,135.49,135.64,139.71,141.94,144.99,146.52,147.50$, 197.73; $\mathrm{UV} \lambda_{\max }=373 \mathrm{~nm}\left(\mathrm{CH}_{2} \mathrm{Cl}_{2}\right) ; \mathrm{PL} \lambda_{\max }=508 \mathrm{~nm}\left(\mathrm{CH}_{2} \mathrm{Cl}_{2}\right)$.

1,3,5-Tris(4'-( $N, N$-bis(3'-methoxybiphenyl-4-yl)amino)biphenyl-4-yl)benzene (4g): It was obtained from DMSO as dark green powder ( $150 \mathrm{mg}, 67 \%$ yield); $\mathrm{mp} 178-179^{\circ} \mathrm{C}$; IR (KBr) v 1599, 1514, 1479, 1320, 1293, 1212, 817, 777, 694, $532 \mathrm{~cm}^{-1}$; ${ }^{1} \mathrm{H}-\mathrm{NMR}\left(300 \mathrm{MHz}, \mathrm{CDCl}_{3}\right) \delta 3.89(\mathrm{~s}, 18 \mathrm{H}), 6.90(\mathrm{~d}, J=8.0 \mathrm{~Hz}$, $6 \mathrm{H}), 7.17-7.28(\mathrm{~m}, 30 \mathrm{H}), 7.37(\mathrm{t}, J=7.9 \mathrm{~Hz}, 6 \mathrm{H}), 7.55(\mathrm{~d}, J=8.5$ $\mathrm{Hz}, 12 \mathrm{H}), 7.61(\mathrm{~d}, J=8.4 \mathrm{~Hz}, 6 \mathrm{H}), 7.73(\mathrm{~d}, J=8.1 \mathrm{~Hz}, 6 \mathrm{H}), 7.81$ $(\mathrm{d}, J=8.1 \mathrm{~Hz}, 6 \mathrm{H}), 7.90(\mathrm{~s}, 3 \mathrm{H}),{ }^{13} \mathrm{C}-\mathrm{NMR}\left(75 \mathrm{MHz}, \mathrm{CDCl}_{3}\right)$ $\delta 55.33,112.32,112.47,119.30,124.47,124.87,127.15,127.76$, $127.88,128.04,129.83,134.95,135.54,139.59,139.81,141.98$, 142.12, 146.91, 146.96, 159.98; UV $\lambda_{\max }=355 \mathrm{~nm}\left(\mathrm{CH}_{2} \mathrm{Cl}_{2}\right)$;
PL $\lambda_{\max }=495 \mathrm{~nm}\left(\mathrm{CH}_{2} \mathrm{Cl}_{2}\right)$.

1,3,5-Tris(4'-( $N, N$-bis(4'-phenoxybiphenyl-4-yl)amino)biphenyl-4-yl)benzene (4h): It was obtained from DMSO as dark green powder (193 mg, 71\% yield); mp $196-198^{\circ} \mathrm{C}$; IR (KBr) v 1589, 1486, 1322, 1238, 1167, 870, 818, 751, 692, 519 $\mathrm{cm}^{-1} ;{ }^{1} \mathrm{H}-\mathrm{NMR}\left(300 \mathrm{MHz}, \mathrm{CDCl}_{3}\right) \delta$ 7.09-7.28 (m, 48H), 7.39 $(\mathrm{t}, J=7.6 \mathrm{~Hz}, 12 \mathrm{H}), 7.52-7.63(\mathrm{~m}, 30 \mathrm{H}), 7.74(\mathrm{~d}, J=7.7 \mathrm{~Hz}$, $6 \mathrm{H}), 7.83(\mathrm{~d}, J=8.1 \mathrm{~Hz}, 6 \mathrm{H}), 7.91(\mathrm{~s}, 3 \mathrm{H}) ; \mathrm{UV} \lambda_{\max }=355 \mathrm{~nm}$ $\left(\mathrm{CH}_{2} \mathrm{Cl}_{2}\right)$; PL $\lambda_{\max }=491 \mathrm{~nm}\left(\mathrm{CH}_{2} \mathrm{Cl}_{2}\right)$.

1,3,5-Tris(4'-( $N, N$-bis(4'-( $N, N$-dimethylamino)biphenyl4-yl)amino)biphenyl-4-yl)benzene (4i): It was obtained from toluene as orange powder $(140 \mathrm{mg}, 60 \%$ yield $) ; \mathrm{mp}>300^{\circ} \mathrm{C}$; IR (KBr) v 3474, 1609, 1497, 1320, $811 \mathrm{~cm}^{-1}$; ${ }^{1} \mathrm{H}-\mathrm{NMR}$ (300 $\left.\mathrm{MHz}, \mathrm{CDCl}_{3}\right) \delta 3.01(\mathrm{~s}, 36 \mathrm{H}), 6.83(\mathrm{~d}, J=7.0 \mathrm{~Hz}, 12 \mathrm{H}), 7.16-$ $7.24(\mathrm{~m}, 18 \mathrm{H}), 7.49-7.60(\mathrm{~m}, 30 \mathrm{H}), 7.72(\mathrm{~d}, J=7.9 \mathrm{~Hz}, 6 \mathrm{H}), 7.80$ $(\mathrm{d}, J=7.9 \mathrm{~Hz}, 6 \mathrm{H}), 7.90(\mathrm{~s}, 3 \mathrm{H}) ;{ }^{13} \mathrm{C}-\mathrm{NMR}\left(75 \mathrm{MHz}, \mathrm{CDCl}_{3}\right)$ $\delta 40.87,113.23,123.72,124.11,124.74,125.02,125.37,127.04$, $127.38,127.70,127.85,132.25,134.19,135.75,139.42,139.93$, $141.99,145.78,146.82,147.30 ; \mathrm{UV} \lambda_{\max }=358 \mathrm{~nm}\left(\mathrm{CH}_{2} \mathrm{Cl}_{2}\right)$; $\mathrm{PL} \lambda_{\max }=500 \mathrm{~nm}\left(\mathrm{CH}_{2} \mathrm{Cl}_{2}\right)$.

Acknowledgments. This research was supported by Leading Technology Development Program funded by Small and Medium Business Administration.

\section{References}

1. Grayson, S. M.; Fréchet, J. M. J. Chem. Rev. 2001, 101, 3819.

2. Fréchet, J. M. J. Science 1994, 263, 1710.

3. Milhem, O. M.; Myles, C.; Mekeown, N. B.; Attwood, D.; Emanuele, A. D. Int. J. Pharm. 2000, 197, 239.

4. Pistolis, G.; Malliaris, A.; Tsiourvas, D.; Paleos, C. M. Chem. Eur. J. 1999, 5, 1440.

5. Kobayashi, H.; Brechbiel, M. W. Adv. Drug Delivery Rev. 2005, $57,2271$.

6. Boas, U.; Heegaard, P. M. H. Chem. Soc. Rev. 2004, 33, 43.

7. Heldt, J. M.; Durand, N. F.; Salmain, M.; Vessieres, A.; Jaouen, G. J. Organomet. Chem. 2004, 689, 4775.

8. Kim, T.-I.; Bai, C. Z.; Park, J.-S. Bull. Kor. Chem. Soc. 2007, 28, 1317.

9. Nostrum, C. F. V. Adv. Drug. Delivery Rev. 2004, 56, 9.

10. Burn, P. L.; Lo, S.-C.; Samuel, D. W. Adv. Mater. 2007, 19, 1675.

11. Hwang, S. H.; Moorefield, C. N.; Newkome, G. R. Chem. Soc. Rev. 2008, 37, 2543.

12. Lee, J. W.; Kim, J. H.; Kim, B.-K.; Kim, J. H.; Shin, W. S.; Jin, S.-H.; Kim, M. Bull. Kor. Chem. Soc. 2006, 27, 1795.

13. Brauge, L.; Magro, G.; Caminade A.-M.; Majoral, J.-P. J. Am. Chem. Soc. 2001, 123, 6698.

14. Goodwin, A. P.; Lam, S. S.; Fréchet, J. M. J. J. Am. Chem. Soc. 2007, 129, 6994 .

15. Bourrier, O.; Kakkar, A. K. J. Mater. Chem. 2003, 6, 1306.

16. Franc, G.; Kakkar, A. K. Chem. Eur. J. 2009, 15, 5630.

17. Killops, K. L.; Campos, L. M.; Hawker, C. J. J. Am. Chem. Soc. 2008, 130, 5062.

18. Lee, J. W.; Kim, B.-K.; Han, S. C.; Kim, J. H. Bull. Kor. Chem. Soc. 2009, 30, 157.

19. Lee, J. W.; Kim, B.-K.; Jin, S.-H. Bull. Kor. Chem. Soc. 2005, 26, 833.

20. Elmorsy, S. S.; Pelter, A.; Smith, K. Tetrahedron Lett. 1991, 32, 4175.

21. Miyaura, N.; Suzuki, A. Chem. Rev. 1995, 95, 2457. 